\title{
Efecto del molibdeno en el frijol común de invierno (Phaseolus vulgaris L.): fertilización foliar y semillas enriquecidas
}

\author{
Effect to molybdenum in the common winter bean (Phaseolus vulgaris L.): \\ foliar fertilization and enriched seeds
}

\author{
Lorena Almeida de Oliveira ${ }^{1}$, Vanderley José Pereira ${ }^{*}$, Marcos Gleidson Pereira dos Santos ${ }^{2}$, \\ Regina Maria Quintão Lana ${ }^{1}$, Ariel Santivañez Aguilar ${ }^{3}$
}

\section{RESUMEN}

El molibdeno (Mo) tiene un papel fundamental en el aumento de la productividad del frijol al potencializar la fijación biológica del nitrógeno. El objetivo de este trabajo fue evaluar la calidad nutricional de cv. Madrepérola, con distintos niveles de molibdeno en las semillas y bajo aplicación foliar de este micronutriente. Para la producción de semillas se fertilizó la "planta madre" con molibdato de sodio en la dosis de $0 ; 300$ y $600 \mathrm{~g} \mathrm{ha}^{-1}$. Con las semillas se instaló un experimento en bloques al azar, con cuatro repeticiones, en esquema factorial 3 x 2: tres niveles de molibdeno en las semillas y dos dosis de molibdeno vía foliar. Se evaluaron los contenidos de $\mathrm{Co}, \mathrm{Fe}, \mathrm{K}, \mathrm{Mo}, \mathrm{N}$ y Zn en las hojas y los granos. La aplicación foliar de molibdeno incrementa los niveles de hierro y molibdeno en las hojas y semillas, y la aplicación de zinc en las hojas. Las semillas enriquecidas con Mo mejoran la nutrición de la planta con incrementos de 8,98 y 7,42 $\mathrm{g} \mathrm{kg}^{-1}$ de nitrógeno en los granos de plantas provenientes de semillas con bajo y medio contenido de molibdeno, respectivamente, y $8,34 \mathrm{mg} \mathrm{dm}^{-3} \mathrm{de} \mathrm{Zn}$ en las hojas de plantas provenientes de semillas con medio contenido de molibdeno.

Palabras clave: micronutriente, nutrición del frijol, actividad enzimática, fijación biológica de nitrógeno, interacción entre nutrientes.

\begin{abstract}
Molybdenum (Mo) has a fundamental role in increasing bean productivity by potentiating the biological fixation of nitrogen. In this sense, the objective was to evaluate the nutritional quality $c v$. Madrepérola, with variations in the levels of molybdenum in the seeds and under foliar application of this micronutrient. For the production of seeds, the "mother plant" was fertilized with sodium molybdate in the dose of 0; 300 and $600 \mathrm{~g} \mathrm{ha}^{-1}$. With the seeds, an experiment was installed in randomized blocks, with four repetitions, in a factorial scheme: three levels of molybdenum in the seeds and two doses of molybdenum via foliar. The contents of $\mathrm{Co}, \mathrm{Fe}, \mathrm{K}, \mathrm{Mo}, \mathrm{N}$ and $\mathrm{Zn}$ in the leaves and grains were evaluated. The foliar application of molybdenum increases the levels of iron and molybdenum in the leaves and seeds and zinc in the leaves. The seeds enriched with Mo improves the nutrition of the plant (increases of 8.98 and $7.42 \mathrm{~g} \mathrm{~kg}^{-1}$ of nitrogen in the grains of plants from seeds with low and medium molybdenum content, respectively: $8.34 \mathrm{mg} \mathrm{dm}-3 \mathrm{Zn}$ in the leaves of plants from seeds with a medium content of molybdenum).
\end{abstract}

Keywords: micronutrient, bean nutrition, enzymatic activity, biological nitrogen fixation, interaction between nutrients.

\section{Introducción}

Brasil es el tercer mayor productor mundial de frijoles (Phaseolus vulgaris L.), con una producción de 3345,5 mil toneladas estimadas para la cosecha 2017/18, con un área plantada que llega a 3185,8 mil hectáreas (CONAB, 2017). Esta producción se concentra en propiedades pequeñas y poco tecnificadas. A ello se suma su importancia social, ya que el frijol es una de las principales fuentes de proteína de la población de bajos ingresos. Además presenta características nutricionales interesantes (Andrade et al., 2004). El frijol es poco consumido en el mercado exterior, y en este sentido, Brasil puede convertirse en un exponente, por producir en tres épocas: cosecha de las aguas,

\footnotetext{
Universidade Federal de Uberlândia - Instituto de Ciências Agrárias - Uberlândia, MG, Brasil.

Universidade Federal de Viçosa - Departamento de Fitotecnia - Viçosa, MG, Brasil.

Universidade Estadual Paulista Júlio de Mesquita Filho - Botucatu, Sao Paulo-Brasil.

* Autor correspondiente: vamceres.vanderley@gmail.com
}

Fecha de Recepción: 25 de marzo, 2020.

Fecha de Aceptación: 6 de junio, 2020. 
de la sequía e irrigada. Para que esta proyección se haga realidad es necesario maximizar el sistema de cultivo, destacando la eficiencia de la fertilización.

En el cultivo del frijol, el nitrógeno (N) es el macronutriente más absorbido y extraído por la planta, lo cual redunda en un aumento de producción y calidad, principalmente el contenido de proteína, nitrógeno, cobre, manganeso y triptófano en los granos (Andrade et al., 2004). Sin embargo, los fertilizantes nitrogenados son de alto costo, y pueden ocurrir pérdidas en el suelo causando daños al ambiente. Por lo tanto, es importante el uso de técnicas que mejoran la eficiencia del uso del $\mathrm{N}$ por la planta, generando un incremento en la productividad, así como menores gastos y problemas ecológicos. Una de esas técnicas es la maximización de la fijación biológica del nitrógeno $(\mathrm{FBN})$.

La FBN es más eficiente con el suministro de molibdeno (Mo) (Leite et al., 2009). El Mo forma parte de las enzimas nitrato reductasa y nitrogenasa involucradas en la fijación de $\mathrm{N}$ (Ferreira et al., 2003). Ccomo están correlacionadas en las mismas rutas fisiológicas, los síntomas de deficiencia del Mo se confunden con N. Los estudios demuestran que la aplicación de Mo actúa en el aumento de la productividad del frijol con la fertilización nitrogenada, pero a veces puede incluso sustituirla, por potenciar la FBN (Amane et al., 1999). El Mo puede ser suministrado a la planta directamente en el suelo, vía foliar o por medio del enriquecimiento de las semillas. Existen cultivares con mayor capacidad de acumulación de Mo en la semilla (Ferreira et al., 2003; Leite et al., 2009).

El uso de semillas enriquecidas con Mo genera beneficios con menores pérdidas del fertilizante y mayor eficiencia del uso del micronutriente, ya que éste será puesto a disposición para la planta de acuerdo con la necesidad (Leite et al., 2009). El contenido de Mo en la semilla aisladamente mejora los procesos fisiológicos (Ferreira et al., 2003; Leite et al., 2009) y asociado a la fertilización vía foliar con Mo aumenta la productividad (Flavio et al., 2004).

Existe escasez de literatura que investiga el enriquecimiento de las semillas con molibdeno (efecto materno), por la vía de la fertilización, cuyas semillas, en sí, son la fuente de suministro nutricional. En este sentido, se buscó corroborar la hipótesis de que la fijación biológica de nitrógeno en plantas de frijol es eficiente cuando los niveles de molibdeno son adecuados y que estos pueden ser suplidos por las reservas (efecto materno). Por lo tanto, este trabajo tuvo como objetivo evaluar la calidad nutricional de la cultivar Madrepérola, producida en el período de invierno con variaciones de los niveles de molibdeno en las semillas y bajo aplicación foliar de este micronutriente.

\section{Material y métodos}

Para la producción de semillas con tres niveles de molibdeno: bajo: cantidad no detectada, medio: 2,3 microgramos por semilla, y alto: 5,2 microgramos por semilla, se fertilizó la planta madre con molibdato de sodio con la dosis de 0 ; 300 y $600 \mathrm{~g} \mathrm{ha}^{-1}$. La fertilización fue parcelada, siendo para la dosis de $300 \mathrm{~g} \mathrm{ha}^{-1}, 100 \mathrm{~g}$ en $\mathrm{V}_{4}$ (tercera hoja trifoliada) y $200 \mathrm{~g}$ en R5 (inicio del florecimiento) y para la dosis de $600 \mathrm{~g} \mathrm{ha}^{-1}, 100 \mathrm{~g}$ en $\mathrm{V}_{4}, 250 \mathrm{~g}$ en $\mathrm{R}_{5}$ y $250 \mathrm{~g}$ en $\mathrm{R}_{7}$ (cuando $50 \%$ de las plantas poseían vaina). Las semillas fueron cedidas por la Universidad Federal de Viçosa a la Universidad Federal de Uberlândia, donde se realizó el experimento en el año 2014, en la hacienda experimental Capim Branco (UFU), Uberlândia-MG (coordenadas geográficas de $18^{\circ}$ $55^{\prime} 23^{\prime \prime}$ latitud sur y $48^{\circ} 17^{\prime} 19^{\prime}$ ' longitud). El clima de Uberlândia, según la clasificación de Kõppen, es del tipo Aw, megatérmico, con lluvias en verano y seca en invierno.

El suelo presenta histórico de rotación de cultivo con soja y maíz por diez años, y es clasificado como Latossolo Vermelho-Amarelo de textura arcillosa. Presenta niveles de $\mathrm{pH}$ y potasio adecuados y de fósforo bajo (Tabla 1; Ribeiro 1999). La fertilización fue realizada con $500 \mathrm{~kg} \mathrm{ha}^{-1}$ del formulado 6-18-6 en la siembra, cuyas fuentes fueron urea $(45 \%$ de $\mathrm{N})$, superfosfato triple $\left(41 \%\right.$ de $\left.\mathrm{P}_{2} \mathrm{O}_{5}\right)$ y cloruro de potasio $\left(60 \%\right.$ de $\left.\mathrm{K}_{2} \mathrm{O}\right)$. El preparado del suelo se restringió a arado y gradeo. Las semillas de la cultivar Madrepérola fueron sembradas con una densidad de 12 semillas por metro. Las parcelas fueron constituidas por cuatro filas, cada una con cuatro metros, espaciadas por $0,5 \mathrm{~m}$. El área útil correspondió a las dos filas centrales, descartándose medio metro de los laterales. En la mitad de las parcelas se aplicó $500 \mathrm{~g} \mathrm{ha}^{-1}$ de molibdeno vía foliar y en la otra mitad no hubo aplicación, siendo consideradas testigos. Las aplicaciones de molibdeno, cuya fuente utilizada fue el molibdato 
Tabla 1. Características químicas de Latossolo Vermelho de textura argilosa $(0-20 \mathrm{~cm})$

\begin{tabular}{|c|c|c|c|c|c|c|}
\hline \multirow{2}{*}{$\mathrm{pH} \mathrm{H} \mathrm{H}_{2} \mathrm{O}$} & P meh-1 & $\mathrm{K}^{+}$ & $\mathrm{Ca}^{2+}$ & $\mathrm{Mg}^{2+}$ & $\mathrm{Al}^{3+}$ & $\mathrm{H}+\mathrm{Al}$ \\
\hline & $\mathrm{mg} \mathrm{dm}-3$ & \multicolumn{5}{|c|}{$\mathrm{Cmol}_{\mathrm{c}} \mathrm{dm}^{-3}$} \\
\hline 5,5 & 4,4 & 0,41 & 2,3 & 0,9 & 0,0 & 4,70 \\
\hline M.O. & C.O. & B & $\mathrm{Cu}$ & $\mathrm{Fe}$ & Mn & $\mathrm{Zn}$ \\
\hline \multicolumn{2}{|c|}{ dag kg-1 } & \multicolumn{5}{|c|}{$\mathrm{mg} \mathrm{dm}^{-3}$} \\
\hline 3,5 & 2,0 & 0,06 & 7,4 & 18 & 9,4 & 1,5 \\
\hline
\end{tabular}

$\mathrm{P}=$ Método Mehlich1, $\mathrm{P}, \mathrm{K}, \mathrm{Na}=\left[\mathrm{HCl} 0,05 \mathrm{~mol} \mathrm{~L}^{-1}+\mathrm{H}_{2} \mathrm{SO}_{4}\right.$ $\left.0,0125 \mathrm{~mol} \mathrm{~L}^{-1}\right], \mathrm{S}^{-\mathrm{SO}_{4}}=[$ Fosfato Monobásico Calcio 0,01 $\left.\mathrm{mol} \mathrm{L}{ }^{-1}\right], \mathrm{Ca}, \mathrm{Mg}, \mathrm{Al}=\left[\mathrm{KCL} 1 \mathrm{~mol} \mathrm{~L}^{-1}\right] / \mathrm{H}+\mathrm{Al}=$ [Solución Tampón SMP a pH 7,5], M.O. = Método Colorimétrico, S.B = Suma de base, $\mathrm{V}=$ Saturación de Base; $\mathrm{T}=\mathrm{CTC}$ pH 7,0; Fuente: Datos obtenidos por medio del trabajo realizado en el Laboratorio de análisis de suelo de la Universidad Federal de Uberlândia (LABAS-UFU) segundo Donagema et al. (2011).

de sodio, se dividieron en dos etapas: la primera en el estadio $\mathrm{V}_{4}$ en la dosis de $100 \mathrm{~g} \mathrm{ha}^{-1}$ diluida en $2400 \mathrm{ml}$ de agua y la segunda en el estadio $\mathrm{R}_{7}$ en la dosis $400 \mathrm{~g} \mathrm{ha}^{-1}$ diluida en $2400 \mathrm{ml}$ de agua. Las aplicaciones se realizaron de forma uniforme con un pulverizador manual, en las cuatro filas, con volumen $200 \mathrm{ml}$ por parcela.

El experimento correspondió a un factorial con 3 niveles de molibdeno en las semillas x 2 dosis de molibdeno vía foliar (0 y $500 \mathrm{~g} \mathrm{ha}^{-1} \mathrm{de}$ molibdato de sodio) con cuatro repeticiones, en delineamiento de bloques completos al azar. Durante el desarrollo del cultivo se realizaron los manejos culturales y fitosanitarios recomendados para esta especie y el riego por aspersión siempre que fue necesario. En la fase de floración $\left(\mathrm{R}_{6}\right)$ se retiraron 10 muestras de hojas de las filas centrales. Estas hojas estaban con máximo crecimiento, pero aún jóvenes (colectadas del tercio medio de las plantas). Las hojas fueron secadas en invernadero para la realización de los análisis de macronutrientes $(\mathrm{N}$ y K) y micronutrientes (Mo, Co, Fe y Zn). Las semillas de las plantas fueron cosechadas y luego secadas (12-13\%, base húmeda). 200 semillas fueron enviadas al laboratorio de análisis de suelos de la Universidad Federal de Viçosa para el análisis de $\mathrm{Co}, \mathrm{Fe}, \mathrm{K}, \mathrm{Mo}$ y $\mathrm{Zn}$ y el restante se usó para el análisis de $\mathrm{N}$ en el Laboratorio de la Universidad Federal de Uberlândia (LABAS -UFU). Todos los análisis se basaron en las recomendaciones de Donagema et al. (2011).

Los datos fueron sometidos al análisis de la normalidad de los residuos y la homogeneidad de las variancias por las pruebas de KolmogorovSmirnov y Levene, respectivamente, ambos a 0,01 de significancia. Cuando al menos una de las presuposiciones no fue atendida, los datos se sometieron a la transformación del tipo raíz cuadrada. Se realizó el análisis de varianza (ANOVA) y las medias fueron comparadas por el test de Tukey, ambas a 0,05 de significancia.

\section{Resultados y discusión}

El uso de semillas enriquecidas con niveles crecientes de molibdeno (Mo) no incrementó los niveles de $\mathrm{N}$ y $\mathrm{K}$ en las hojas (Tabla 2) y de K, $\mathrm{Zn}$ y Co en los granos (Tablas 4 y 5). Tampoco la aplicación foliar con dosis de $500 \mathrm{~g} \mathrm{ha}^{-1}$ dividida

Tabla 2. Contenidos foliares de macronutrientes de plantas provenientes de semillas con diferentes contenidos de molibdeno y aplicación foliar.

\begin{tabular}{|c|c|c|c|c|c|c|}
\hline \multirow{3}{*}{$\begin{array}{l}{ }^{1} \text { Contenido de } \\
\text { Mo en semillas }\end{array}$} & \multicolumn{3}{|c|}{ Nitrógeno $\left(\mathrm{g} \mathrm{kg}^{-1}\right)$} & \multicolumn{3}{|c|}{ Potasio $\left(\mathrm{mg} \mathrm{dm}^{-3}\right)$} \\
\hline & \multicolumn{2}{|c|}{ Dosis de Mo foliar } & \multirow{2}{*}{ Media } & \multicolumn{2}{|c|}{ Dosis de Mo foliar } & \multirow{2}{*}{ Media } \\
\hline & 0 & 500 & & 0 & 500 & \\
\hline Bajo & 40,91 & 45,02 & $42,96 \mathrm{a}$ & 82,25 & 92,75 & $87,5 \mathrm{a}$ \\
\hline Medio & 43,90 & 39,64 & $41,77 \mathrm{a}$ & 99,5 & 92,5 & $96,0 \mathrm{a}$ \\
\hline Alto & 41,65 & 40,19 & $40,92 \mathrm{a}$ & 81,5 & 83,25 & $82,38 \mathrm{a}$ \\
\hline Media & $42,15 \mathrm{~A}$ & $41,62 \mathrm{~A}$ & & $87,75 \mathrm{~A}$ & $89,5 \mathrm{~A}$ & \\
\hline
\end{tabular}

${ }^{2} F^{\prime}(P) ; W(P) \quad 2,937(0,041) ; 0,987(0,983) \quad 0,491(0,779) ; 0,944(200)$

${ }^{1}$ Medias seguidas de letras distintas, mayúsculas en la línea y minúscula en la columna difieren entre sí por la prueba de Tukey a 0,05 de significancia.

${ }^{2} \mathrm{~F}(\mathrm{P})$; W(P): Estadística y probabilidad de la prueba de Levene y Shapiro-Wilk, respectivamente. Probabilidades mayores que 0,01 indican homocedasticidad y adherencia a Normal, respectivamente. 
en $V_{4}$ y $R_{7}\left(100\right.$ y $400 \mathrm{~g} \mathrm{ha}^{-1}$, respectivamente) alteró los valores. En resumen, el manejo del Mo no tuvo impacto en los contenidos de estos nutrientes, quedando cerca de $40 \mathrm{~g} \mathrm{~kg}^{-1}$ y $85 \mathrm{mg}$ $\mathrm{dm}^{-3}$ para $\mathrm{N}$ y $\mathrm{K}$ en las hojas, respectivamente (Tabla 2), y 8,5; 31,5 y $2,0 \mathrm{mg} \mathrm{dm}^{-3}$ para $\mathrm{K}, \mathrm{Zn}$ y Co en los granos (Tablas 4 y 5 ).

La combinación entre los niveles endógenos y la aplicación foliar de Mo influyó en los micronutrientes foliares, a excepción del Co (Tabla 3). Para este micronutriente, los valores obtenidos fueron bajos, y no llegaron a $1 \mathrm{mg}$ $\mathrm{dm}^{-3}$ (Tabla 3). Solamente las plantas oriundas de semillas con alto contenido de Mo fueron sensibles a la aplicación foliar, resultando en aumento en los contenidos foliares de Fe. El incremento de los niveles endógenos de Mo en las semillas no se reflejó en mayores contenidos de Fe foliar. Al realizar la aplicación foliar de Mo en plantas oriundas de semillas con altos contenidos de este nutriente, el aumento de Fe fue significativo (2023,72 $\mathrm{mg} \mathrm{dm}^{-3}$ ); sin embargo, no difirió de las semillas con bajos contenidos (Tabla 3 ).
Los niveles foliares de $\mathrm{Zn}$ fueron superiores en plantas oriundas de semillas con niveles medios de Mo, pero al efectuar la fertilización foliar, el impacto de los contenidos endógenos de molibdeno resultó irrelevante (Tabla 3). La aplicación foliar de Mo incrementó los niveles de $\mathrm{Zn}$ en plantas en las que el aporte de Mo de las semillas fue bajo y en las plantas provenientes de semillas con medios contenidos, la fertilización foliar resultó en menores niveles de $\mathrm{Zn}\left(18,61 \mathrm{mg} \mathrm{dm}^{-3}\right)$ (Tabla 3). Para los contenidos de Mo foliares no hubo efecto de las diferentes concentraciones de este nutriente en las semillas, pero al realizar la aplicación foliar, las plantas originadas de semillas con niveles medios y altos fueron las que tuvieron resultados inferiores $\left(33,02\right.$ y $22,70 \mathrm{mg} \mathrm{dm}^{-3}$, respectivamente). Sin embargo, la fertilización foliar es esencial, ya que para todos los niveles de Mo en las semillas, la aplicación foliar resultó en incrementos (Tabla 3). En este contexto el menor aumento fue para las semillas con altos contenidos de Mo (22,31 mg dm ${ }^{-3}$ en las hojas) (Tabla 3). Para granos el resultado fue aún más notorio,

Tabla 3. Contenidos de micronutrientes foliares de plantas provenientes de semillas con contenidos distintos de molibdeno y aplicación foliar.

\begin{tabular}{|c|c|c|c|c|c|c|}
\hline \multirow{3}{*}{$\begin{array}{l}\text { Contenido de Mo } \\
\text { en la semilla }\end{array}$} & \multicolumn{3}{|c|}{ Zinc $\left(\mathrm{mg} \mathrm{dm}^{-3}\right)$} & \multicolumn{3}{|c|}{ Molibdeno $\left(\mathrm{mg} \mathrm{dm}^{-3}\right)$} \\
\hline & \multicolumn{2}{|c|}{ Dosis de Mo foliar } & \multirow{2}{*}{ Media } & \multicolumn{2}{|c|}{ Dosis de Mo foliar } & \multirow{2}{*}{ Media } \\
\hline & 0 & 500 & & 0 & 500 & \\
\hline Bajo & $18,05 \mathrm{bB}$ & $22,36 \mathrm{aA}$ & 20,21 & $0,05 \mathrm{aB}$ & $42,83 \mathrm{aA}$ & 21,44 \\
\hline Medio & $26,39 \mathrm{aA}$ & $18,61 \mathrm{aB}$ & 22,50 & $0,04 \mathrm{aB}$ & $33,02 \mathrm{bA}$ & 16,53 \\
\hline Alto & $18,77 \mathrm{bA}$ & $19,99 \mathrm{aA}$ & 19,38 & $0,39 \mathrm{aB}$ & $22,70 \mathrm{cA}$ & 11,54 \\
\hline \multirow[t]{2}{*}{${ }^{2} F^{\prime}(P) ; W(P)$} & \multicolumn{3}{|c|}{$2,667(0,057) ; 0,976(0,811)$} & \multicolumn{3}{|c|}{$1,292(0,311) ; 0,904(0,026)$} \\
\hline & \multicolumn{3}{|c|}{ Hierro $\left(\mathrm{mg} \mathrm{dm}^{-3}\right)$} & \multicolumn{3}{|c|}{ Cobalto $\left(\mathrm{mg} \mathrm{dm}^{-3}\right)$} \\
\hline Bajo & $1335,40 \mathrm{aA}$ & $1644,83 \mathrm{abA}$ & 1491,61 & 0,48 & 0,58 & $0,53 \mathrm{a}$ \\
\hline Medio & $1578,06 \mathrm{aA}$ & $1233,52 \mathrm{bA}$ & 1405,79 & 0,55 & 0,50 & $0,53 \mathrm{a}$ \\
\hline Alto & $1194,35 \mathrm{aB}$ & $2023,72 \mathrm{aA}$ & 1609,04 & 0,53 & 0,61 & $0,57 \mathrm{a}$ \\
\hline Media & 1369,27 & 1635,02 & & $0,52 \mathrm{~A}$ & $0,56 \mathrm{~A}$ & \\
\hline \multirow[t]{2}{*}{${ }^{2} F^{\prime}(P) ; W(P)$} & \multicolumn{3}{|c|}{$1,811(0,161) ; 0,967(0,592)$} & \multicolumn{3}{|c|}{$8,883(<0,001) ; 0,970(0,662)$} \\
\hline & \multicolumn{3}{|c|}{$\operatorname{Zinc}\left(\mathrm{mg} \mathrm{dm}^{-3}\right)$} & \multicolumn{3}{|c|}{ Molibdeno $\left(\mathrm{mg} \mathrm{dm}^{-3}\right)$} \\
\hline Bajo & $18,05 \mathrm{bB}$ & $22,36 \mathrm{aA}$ & 20,21 & $0,05 \mathrm{aB}$ & $42,83 \mathrm{aA}$ & 21,44 \\
\hline Medio & $26,39 \mathrm{aA}$ & $18,61 \mathrm{aB}$ & 22,50 & $0,04 \mathrm{aB}$ & $33,02 \mathrm{bA}$ & 16,53 \\
\hline Alto & $18,77 \mathrm{bA}$ & 19,99 aA & 19,38 & $0,39 \mathrm{aB}$ & $22,70 \mathrm{cA}$ & 11,54 \\
\hline${ }^{2} F^{\prime}(P) ; W(P)$ & \multicolumn{3}{|c|}{$2,667(0,057) ; 0,976(0,811)$} & \multicolumn{3}{|c|}{$1,292(0,311) ; 0,904(0,026)$} \\
\hline
\end{tabular}

${ }^{1}$ Medias seguidas de letras distintas, mayúsculas en la línea y minúscula en la columna por la prueba de Tukey a 0,05 de significancia.

${ }^{2} \mathrm{~F}$ '(P); W (P): Estadística y probabilidad de la prueba de Levene y Shapiro-Wilk, respectivamente. Las probabilidades mayores que 0,01 indican homocedasticidad y adherencia a Normal, respectivamente. 
Tabla 4. Contenido de macronutrientes en las semillas oriundos de plantas de frijol provenientes de semillas con distintos contenidos de molibdeno y aplicación foliar.

\begin{tabular}{|c|c|c|c|c|c|c|}
\hline \multirow{3}{*}{$\begin{array}{l}{ }^{1} \text { Contenido de Mo } \\
\text { en la semilla }\end{array}$} & \multicolumn{3}{|c|}{ Nitrógeno $\left(\mathrm{g} \mathrm{kg}^{-1}\right)$} & \multicolumn{3}{|c|}{ Potasio $\left(\mathrm{mg} \mathrm{dm}^{-3}\right)$} \\
\hline & \multicolumn{2}{|c|}{ Dosis de Mo foliar } & \multirow{2}{*}{ Media } & \multicolumn{2}{|c|}{ Dosis de Mo foliar } & \multirow{2}{*}{ Medi: } \\
\hline & 0 & 500 & & 0 & 500 & \\
\hline Bajo & 52,18 aA & $43,20 \mathrm{aB}$ & 47,69 & 8,76 & 8,85 & $8,80 \mathrm{a}$ \\
\hline Medio & $50,54 \mathrm{aA}$ & $43,12 \mathrm{aB}$ & 46,83 & 8,77 & 8,19 & $8,48 \mathrm{a}$ \\
\hline Alto & $41,11 \mathrm{bA}$ & $47,20 \mathrm{aA}$ & 44,16 & 8,26 & 8,66 & $8,46 \mathrm{a}$ \\
\hline Media & 47,95 & 44,51 & & $8,6 \mathrm{~A}$ & $8,57 \mathrm{~A}$ & \\
\hline${ }^{2} F^{\prime}(P) ; W(P)$ & \multicolumn{3}{|c|}{$0,522(0,756) ; 0,951(0,280)$} & \multicolumn{3}{|c|}{$2,212(0,098) ; 0,962(0,471)$} \\
\hline
\end{tabular}

${ }^{1}$ Medias seguidas de letras distintas, mayúsculas en la línea y minúscula en la columna por la prueba de Tukey a 0,05 de significancia. ${ }^{2} \mathrm{~F}$ '(P); W (P): Estadística y probabilidad de la prueba de Levene y ShapiroWilk, respectivamente. Las probabilidades mayores que 0,01 indican homocedasticidad y adherencia a Normal, respectivamente.

Tabla 5. Contenidos en las semillas de micronutrientes provenientes de plantas de frijol provenientes de semillas con distintos contenidos de molibdeno y aplicación foliar.

\begin{tabular}{|c|c|c|c|c|c|c|}
\hline \multirow{3}{*}{$\begin{array}{l}{ }^{1} \text { Contenido de Mo } \\
\text { en la semilla }\end{array}$} & \multicolumn{3}{|c|}{ Zinc $\left(\mathrm{mg} \mathrm{dm}^{-3}\right)$} & \multicolumn{3}{|c|}{ Molibdeno (mg dm-3) } \\
\hline & \multicolumn{2}{|c|}{ Dosis de Mo foliar } & \multirow{2}{*}{ Media } & \multicolumn{2}{|c|}{ Dosis de Mo foliar } & \multirow{2}{*}{ Media } \\
\hline & 0 & 500 & & 0 & 500 & \\
\hline Bajo & 30,40 & 33,80 & $31,52 \mathrm{a}$ & 0,02 & 3,14 & $1,58 \mathrm{a}$ \\
\hline Medio & 30,19 & 34,79 & 32,49 a & 0,06 & 3,26 & $1,66 \mathrm{a}$ \\
\hline Alto & 31,83 & 31,21 & $32,10 \mathrm{a}$ & 0,10 & 3,08 & $1,59 \mathrm{a}$ \\
\hline Media & $30,81 \mathrm{~A}$ & $33,27 \mathrm{~A}$ & & $0,06 \mathrm{~B}$ & $3,16 \mathrm{~A}$ & \\
\hline \multirow[t]{2}{*}{${ }^{3} F^{\prime}(P) ; W(P)$} & \multicolumn{3}{|c|}{$1,728(0,179) ; 0,970(0,663)$} & \multicolumn{3}{|c|}{$7,358(0,001) ; 0,975(0,780)$} \\
\hline & \multicolumn{3}{|c|}{ Hierro $\left(\mathrm{mg} \mathrm{dm}^{-3}\right)$} & \multicolumn{3}{|c|}{ Cobalto $\left(\mathrm{mg} \mathrm{dm}^{-3}\right)$} \\
\hline Bajo & $121,58 \mathrm{aB}$ & $196,35 \mathrm{aA}$ & 158,96 & 2,00 & 1,81 & $1,90 \mathrm{a}$ \\
\hline Medio & $126,30 \mathrm{aA}$ & $144,54 \mathrm{bA}$ & 135,42 & 2,26 & 2,11 & $2,18 \mathrm{a}$ \\
\hline Alto & $101,57 \mathrm{aA}$ & $110,20 \mathrm{bA}$ & 105,88 & 1,74 & 1,66 & $1,70 \mathrm{a}$ \\
\hline Media & 116,48 & 150,36 & & $2,00 \mathrm{~A}$ & $1,86 \mathrm{~A}$ & \\
\hline${ }^{3} F^{\prime}(P) ; W(P)$ & \multicolumn{3}{|c|}{$2,212(0,098) ; 0,962(0,471)$} & \multicolumn{3}{|c|}{$7,358(0,001) ; 0,975(0,780)$} \\
\hline
\end{tabular}

${ }^{1}$ Medias seguidas de letras distintas, mayúsculas en la línea y minúscula en la columna por la prueba de Tukey a 0,05 de significancia.

${ }^{2}$ F '(P); W (P): Estadística y probabilidad de la prueba de Levene y Shapiro-Wilk, respectivamente. Las probabilidades mayores que 0,01 indican homocedasticidad y adherencia a Normal, respectivamente.

pues independiente del contenido de Mo en las semillas, solamente la aplicación foliar redundó en un incremento de 3,10 $\mathrm{mg} \mathrm{dm}^{-3}$ (Tabla 5).

Se observó un comportamiento similar para los niveles de Fe en los granos. Cuando las semillas tenían bajos niveles de Mo la aplicación foliar resultó en incrementos (pasando de 121,58 a 196,35 $\mathrm{mg} \mathrm{dm}^{-3}$ ) (Tabla 5). Se concluye que la concentración era baja en las semillas. Por otro lado, el aumento endógeno de Mo en las semillas asociado a la aplicación foliar derivó en disminución de los contenidos en los granos, lo que puede ser un efecto tóxico por exceso. Igualmente, la acumulación de $\mathrm{N}$ en los granos fue influenciada por la combinación del aporte de Mo proveniente de las semillas y de la aplicación foliar (Tabla 4).

Altos contenidos de Mo fueron deletéreos para los niveles de $\mathrm{N}$ en los granos. Esto se confirmó en dos momentos distintos. En primer lugar, las plantas provenientes de semillas con alto contenido 
de Mo resultaron en menor contenido de $\mathrm{N}$ que las provenientes de semillas con medios y bajos contenidos de molibdeno $\left(52,18\right.$ y $50,54 \mathrm{~g} \mathrm{~kg}^{-1}$, respectivamente). En segundo lugar, fijando los contenidos de Mo, la aplicación foliar de este nutriente en la mayoría de los casos derivó en niveles menores de N (Tabla 4). Todos estos resultados coinciden para la hipótesis de que la diferencia entre cantidad ideal y deletérea de Mo es ligera. El Mo debe ser manejado para suplir la demanda del cultivo y maximizar la absorción y fijación de N. Por lo tanto, tecnologías como la aplicación foliar o el suministro del Mo por las reservas de las semillas ganan importancia en suelos con alta acidez. Los suelos tropicales, generalmente, presentan deficiencia de $\mathrm{N}$ debido al alto intemperismo y por ser pobres en materia orgánica (Cameron, Di y Moir, 2013). En pH cercano a 4, en suelos tropicales, el Mo está adsorbido, siendo común la deficiencia incluso con la presencia del nutriente en el suelo. Además, cuando el Mo se aplica a través del suelo puede ser adsorbido por arcillas y óxidos de $\mathrm{Fe}, \mathrm{Al}$ y $\mathrm{Mn}$, entre otros elementos (Silva et al., 2012; Xu et al., 2013).

Esta condición puede limitar la producción del frijol, por restringir la FBN, ya que el $\mathrm{N}$ es uno de los nutrientes más requeridos por el cultivo. En este sentido, el enriquecimiento de semillas de frijol con Mo culmina en diversas ventajas, pues mejora el aprovechamiento de éste y otros nutrientes por la planta. El uso de estas semillas evita que el Mo se pierda en el momento de la aplicación del fertilizante y pone a disposición el nutriente de acuerdo con la demanda de la planta. El manejo de la fertilización con Mo en el frijol resulta en niveles distintos de los nutrientes en las semillas y hojas. La aplicación foliar de Mo derivó en el aumento de los contenidos foliares de Fe. Uno de los motivos es que la bacteria noduladora es i???????siderófora???, es decir, ¿??bacterias??? que desempeñan la función de secuestrar y transportar hierro $\left(\mathrm{Fe}^{3+}\right.$ del suelo que no está disponible en la planta) en situaciones de deficiencia, y por medio de la excreción de sustancias quelantes tornan el elemento disponible (Benite et al., 2002). Además, el aporte extra de hierro culminó posiblemente en la mejora de dos procesos fundamentales: el fotosintético, pues el Fe es transportador de electrones a través de la ferredoxina en el fotosistema I; y el de FBN a través de FeMoco.
La siembra de semillas con bajos y medianos contenidos de Mo facilitó la absorción de N. Se sabe que la planta necesita pequeñas dosis de Mo, ya que dosis superiores pueden ser deletéreas. La absorción del $\mathrm{N}$ puede ser atmosférica $\left(\mathrm{N}_{2}\right)$, en la cual es absorbido y transformado en $\mathrm{NH}_{3}$ por las bacterias simbióticas; o por medio de la absorción de $\mathrm{NO}^{3-}$ del suelo (Fagan et al., 2007). En estos procesos el Mo actúa como un cofactor de las enzimas nitrogenasa y nitrato reductasa (Leite et al., 2009; Silva et al., 2012; Lopes et al., 2016). Para la primera enzima, el Mo está ligado a un complejo de hierro-azufre y homocitrato, y recibe el nombre de FeMoco (Almeida et al., 2014), el cual actúa en los nódulos radiculares, catalizando la reacción que transforma el $\mathrm{N}_{2}$ en $\mathrm{NH}_{3}$. Cuando la planta absorbe el $\mathrm{N}$ del suelo en forma de nitrato $\left(\mathrm{NO}^{3-}\right)$, el compuesto no está asimilándolo, y exige que sea transformado en $\mathrm{NH}^{4+}$. La primera etapa de esta transformación es la reducción de nitrato a nitrito $\left(\mathrm{NO}_{2}-\right)$, reacción catalizada por la nitrato reductasa. Esta enzima depende, además del cofactor formado por Mo ligado a una pterina (Moco), de la heme y FAD como grupos prostéticos, y que para ejercer la actividad enzimática precisa específicamente de NADH o NADPH (Almeida et al., 2014). La FBN por el frijol es considerada baja, por lo que la necesidad de Mo está más relacionada con la actividad de la nitrato reductasa (Biscaro et al., 2011; Silva et al., 2012).

Sin embargo, el uso de semillas enriquecidas y la aplicación foliar de Mo tienden a aumentar los niveles de $\mathrm{N}$ en las plantas (Bertoldo et al., 2015). Estos no se alteraron con la aplicación de Mo vía foliar asociada al uso de semillas enriquecidas. Lo mismo ocurre para los contenidos de Co y K en las semillas y hojas y de $\mathrm{Zn}$ en las semillas de frijol. Los contenidos de $\mathrm{N}$ y $\mathrm{K}$ foliar no se alteraron con la fertilización molibídica cuando la influencia del $\mathrm{N}$ y del Mo fue evaluada sobre el frijol común cv. Ouro vermelho (Lopes et al., 2014). La fertilización foliar de Mo no alteró los contenidos foliares de K y Zn (Silva et al., 2012; Lopes et al., 2016). A este hecho se atribuye la posible dilución de los nutrientes debido al mayor crecimiento de las plantas, o translocación de este nutriente durante la formación de las semillas. El mayor crecimiento de las plantas puede ser debido al incremento de auxinas liberadas por el simbionte al frijol (Pessoa et al., 2000; Ferreira, et al., 2002). 
Con relación a los contenidos de Mo en el suelo, es posible inferir que no estaban adecuados para el frijol, ya que el aumento de los niveles endógenos del nutriente en las semillas o la aplicación foliar resultaron en incremento de $\mathrm{Fe}$ y Mo en las semillas y hojas, y de Zn en las hojas del frijol. Analizando el efecto del molibdeno sobre el desarrollo y composición mineral del frijol, se observa que los contenidos de Mo en las hojas y semillas de frijol a partir de la fertilización foliar con este nutriente fueron mayores (Pessoa et al., 2000; Silva et al., 2012; Lopes et al., 2014, 2016). Este resultado puede ser debido a la ley del mínimo, según la cual un nutriente en menor cantidad determina el desarrollo del cultivo, aunque los otros nutrientes estén en cantidades adecuadas, y por encima de éstas puede incluso causar daño a la planta. Esto fue notorio al combinar los contenidos relevantes de Mo en las semillas con aplicación foliar del nutriente, lo que redujo el contenido foliar de éste en las plantas y también los niveles de $\mathrm{N}$ en los granos. Por tanto, cuanto mayor sea el contenido de molibdeno en la semilla, menor será la necesidad de aplicación foliar de este nutriente. Estos resultados confirman que los micronutrientes son requeridos en pequeñas cantidades por la planta, dentro de una concentración considerada ideal. Como se sabe, la asimilación de nutrientes por la planta ocurre de acuerdo con la necesidad del cultivo, y luego, en determinado momento, no habrá incrementos incluso en condiciones de alta disponibilidad del nutriente. La no interacción entre el uso de semillas enriquecidas y aplicación foliar de molibdeno para los contenidos de este nutriente en los granos revela que los contenidos presentes en las semillas probablemente se utilizan para el crecimiento y desarrollo de la planta, mientras que los disponibles por la aplicación foliar se usan para la nutrición de los granos.

Altos niveles de Mo en las semillas, por sí solos, son suficientes para potenciar la absorción del Zn. Dos hipótesis se plantean para explicar el incremento: la primera es que el equilibrio nutricional entre los nutrientes proporcionó los aumentos; y la segunda, la absorción de Zn se incrementa con la disminución del pH. Esta merma de $\mathrm{pH}$ en la región de la rizosfera es debido a la reacción para la captación del $\mathrm{N}_{2}$ por la $\mathrm{FBN}$ (mejorada por el molibdeno), en la cual ocurre la liberación de hidrógeno, acidificando el medio y mejorando con ello la absorción del Zn (Macedo y Jacob Neto, 2017). Por último, los contenidos nutricionales foliares evaluados en el estadio vegetativo $R_{6}$ no fueron capaces de pronosticar los contenidos finales de los nutrientes en las semillas (Tablas 2, 3, 4 y 5), pues la absorción ocurre incluso en etapas más avanzadas y la planta drena todas las reservas en la producción de descendientes (Pegoraro, Oliveira, Moreira, Kondo y Portugal, 2014) sin que haya relación directa y proporcional.

\section{Conclusión}

La aplicación foliar de molibdeno es una técnica viable desde el punto de vista fisiológico para la obtención de incrementos de absorción de hierro y molibdeno en las hojas y granos, y de zinc solamente en las hojas del frijol. El uso de semillas enriquecidas con molibdeno debe considerarse como alternativa a la fertilización convencional, ya que los contenidos de este nutriente en las semillas pueden ser suficientes para mejorar la nutrición de la planta (incrementos de 8,98 y $7,42 \mathrm{~g} \mathrm{~kg}^{-1}$ de nitrógeno en el caso de las semillas con un contenido medio de molibdeno, y $8,34 \mathrm{mg}$ $\mathrm{dm}^{-3}$ de $\mathrm{Zn}$ en las hojas de plantas procedentes de semillas con medio contenido de molibdeno), dispensando la aplicación foliar.

La diferencia entre el contenido de molibdeno ideal para cultivo y aquel causante de toxicidad es sutil, pues hubo la interacción entre los niveles de molibdeno en las semillas (de cantidad no detectada a 5,2 $\mu$ g semillas $^{-1}$ ) y la dosis foliar (de 0 , y $500 \mathrm{~g} \mathrm{ha}^{-1}$ ) para nitrógeno en los granos y de molibdeno en las hojas. Además, la absorción de molibdeno ocurre en cada una de las etapas de la planta del frijol y existe un drenaje de todas las reservas para producción de descendientes. 


\section{Literatura Citada}

Almeida, J.L.; Carvalho, M.A.C.; Yamashita, O.M.; Silva, A.F.; Teixeira A.O.

2014. Adubação nitrogenada em cobertura e aplicação foliar de cobalto e molibdênio na cultura do feijão. Revista de Ciências Agroambientais, 12: 125-132.

CONAB.

2017. Acompanhamentos da safra brasileira: Grãos. Companhia Brasileira de abastecimento. 145 p.

Amane, M.I.V.; Vieira, C.; Novais, R.F.; Araújo, G.A.A. 1999. Adubação nitrogenada e molíbdica da cultura do feijão na Zona da Mata de Minas Gerais. Revista Brasileira de Ciência do Solo, 23(3): 643-650.

Andrade, C.A.B.; Patroni, S.M.S.; Clemente, E. y Scapim, C.A. 2004. Produtividade e qualidade nutricional de cultivares de feijão em diferentes adubações. Ciência e Agrotecnologia, 28(5):1077-1086.

Benite, A.M.C.; Machado, S.P.; Machado, B.C. 2002. Sideróforos: uma resposta dos microorganismos. Química Nova, 25 (6b): 1155-1164.

Bertoldo, J.G.; Pelisser, A.; Silva, R.P.; Favreto, R.; Oliveira, L.A.D.

2015. Alternativas na fertilização de feijão visando a reduzir a aplicação de N-ureia1. Pesquisa Agropecuária Tropical, 45(3): 348-355

Biscaro, G.A.; Freitas Junior, N.A.; Soratto, R.P.; Kikuti, H.; Goulart Junior, S.A.R.; Aguirre, W.M.

2011. Nitrogênio em cobertura e molibdênio via foliar no feijoeiro irrigado cultivado em solo de cerrado. Acta Scientiarum. Agronomy, 33(4): 665-670.

Cameron, K.C.; Di, H.J.; Moir, J.L.

2013. Nitrogen losses from the soil/plant system: a review. Annals of Applied Biology, 162(2): 145-173.

Donagema, G.K.; Campos, D.V.B.; Calderano, S.B.; Teixeira, W.G.; Vieira, J.H.M.

2011. Manual de métodos de análises de solo. 2 ed. Embrapa Solos. Rio de Janeiro, Brasil. 230 p.

Fagan, E.B.; Medeiros, S.L.P.; Manfron, P.A.; Casaroli, D.; Simon, J.; Dourado Neto, D.; Van Lier, Q.J.; Santos, O.S.; Müller, L.

2007. Fisiologia da fixação biológica do nitrogênio em soja. Revista da faculdade de zootecnia, veterinária e agronomia, 14(1): 89-106.

Ferreira, A.C.B.; Araujo, G.A.A.; Cardoso, A.A.; Fontes, P.C.R.; Vieira, C.

2002. Influência do molibdênio contido na semente e da sua aplicação foliar sobre a composição mineral de folhas e sementes do feijoeiro. Revista Ceres. 49(284): 443-452.

Ferreira, A.C.B.; Araújo, G.A.A.; Cardoso, A.A.; Fontes, P.C.R.; Vieira, C.

2003. Características agronômicas do feijoeiro em função do molibdênio contido na semente e da sua aplicação via foliar. Acta Scientiarum: Agronomy, 25(1): 65-72.
Leite, U.T.; Araújo, G.A.A.; Miranda, G.V.; Vieira, R.F.; Pires, A.A.

2009. Influência do conteúdo de molibdênio na qualidade fisiológica da semente de feijão: cultivares Novo Jalo e Meia Noite. Revista Ceres, 56(2): 225-231.

Lopes, J.F.; Coelho, F.C.; Rabello, W.S.; Rangel, O.J.P.; Gravina, G.A.; Vieira, H.D.

2016. Produtividade e composição mineral do feijão em resposta às adubações com molibdênio e níquel. Revista Ceres, 63(3): 419-426.

Lopes, J.F.; Coelho, F.C.; Rangel, O.J.P.; Rabello, W.S.; Gravina, G.A.; Vieira, H.D.

2014. Adubação foliar com níquel e molibdênio no feijoeiro comum cv. Ouro Vermelho. Revista Ceres, 61 (2): 234-240.

Macedo, R.A.T. y Jacob Neto, J.

2017. Efeitos do nitrato e amônio aplicados via foliar sobre a extrusão de $\mathrm{H}^{+} / \mathrm{OH}^{-}$na rizosfera e na nodulação do feijoeiro (Phaseolus vulgaris). Revista Semioses, 11(1): 57-69.

Pegoraro, R.F.; Oliveira, D.; Moreira, C.G.; Kondo, M.K.; Portugal, A.F.

2014. Partição de biomassa e absorção de nutrientes pelo feijoeiro comum. Revista Caatinga, 27(3): 41-52.

Pessoa, A.C.S.; Ribeiro, A.C.; Chagas, J.M.; Cassini. S.T. A. 2000. Concentração foliar de molibdênio e exportação de nutrientes pelo feijoeiro "ouro negro" em resposta à adubação foliar com molibdênio. Revista Brasileira de Ciências de Solo, 24(1): 75-84.

Possenti, J.C.; Villela, F.A.

2010. Efeito do molibdênio aplicado via foliar e via sementes sobre o potencial fisiológico e produtividade de sementes de soja. Revista Brasileira de Sementes, 32(4): 143-150.

Flavio, J.; Wruck, F.J.; Cobucci, T.; Stone, L.F.

2004. Efeito do tratamento de sementes e da adubação foliar com micronutrientes na produtividade do feijoeiro. Congresso Nacional de Pesquisa de Feijão, 8., 2005, Goiânia. Anais. Embrapa Arroz e Feijão. Goiás, Brasil. pp. 995-998.

Ribeiro, A.C.; Guimarães, P.T.G.; Alvarez V., V.H. 1999. Recomendações para uso de corretivos e fertilizantes em Minas Gerais. 5a aproximação. Comissão de Fertilidade do Solo do Estado de Minas Gerais. Viçosa, Brasil. 359 p.

Silva, E.B.; Santos, S.R.; Fonseca, F.G.; Tanure, L.P.P. y Freitas, J.P.X

2012. Aplicação foliar de molibdênio em feijoeiro irrigado cultivado no norte de Minas Gerais. Bioscience Journal. 28(1): 64-71.

Xu, N.; Braida, W.; Christodoulatos, C. y Chen, J.

2013. A Review of Molybdenum Adsorption in Soils/ Bed Sediments: Speciation, Mechanism, and Model Applications. Soil Sediment Contamination: An International Journal, 22(8): 912-929. 